\title{
Erratum
}

\section{Synthesis of Olefinic Crown Diamides and their Conversion into Pyrazolino Macrocycles: Promising Photoluminescent Crown Compounds}

Rana N. Malhas, Yehia A. Ibrahim* Synthesis 2006, 3261.

The advanced online publication (e-First) version of this article included incorrect author names in reference 12 . This error has been corrected for both the print and current online versions. 